\title{
Revisiting the OZI-forbidden Radiative Decays of Orthoquarkonia*
}

\author{
Gang $\mathrm{Li}^{1}$, Tong $\mathrm{Li}^{2}$, Xue-Qian $\mathrm{Li}^{2}$, Wen-Gan $\mathrm{Ma}^{1}$ and Shu-Min Zhao ${ }^{2}$ \\ ${ }^{1}$ Modern Physics Department, University of Science and Technology of China \\ (USTC), Hefei, Anhui 230026 China \\ 2 Department of Physics, Nankai University, Tianjin 300071, China
}

\begin{abstract}
It is interesting to investigate the OZI-forbidden radiative decays of orthoquarkonium, $J / \psi \rightarrow \gamma \pi^{0}, \gamma \eta$ and $\gamma \eta^{\prime}$ in perturbative QCD. In this work without the approximations adopted in literature we carry out a full one-loop calculation which involves integrations of 4-point and 5-point loop functions. Our numerical results are in agreement with the present data. We also briefly discuss the decays of $J / \psi \rightarrow \gamma+\rho^{0}$, as well as $\Upsilon \rightarrow \pi^{0} \gamma, \eta \gamma, \eta^{\prime} \gamma$.
\end{abstract}

\section{Introduction}

The OZI rule 1 plays an important role in the processes which occur via strong interaction and in general the concerned calculations are carried out in the framework of perturbative QCD. Thus careful studies on such processes where the OZI rule applies can deepen our insight to the perturbative QCD. On other aspect, we would encounter another serious problem. Namely, even though we can accurately calculate the processes at quark-gluon level in terms of perturbative QCD, for evaluating the transition matrix elements, the hadronization which is related to the non-perturbative QCD, must be dealt with. Because of lack of solid knowledge on the non-perturbative QCD, for a whole calculation, one has to employ some model-dependent wavefunctions, which may contaminate the theoretical results. Thus to reduce theoretical uncertainties, the wavefunctions should be well tested and theoretically studied. Then, although the results have more or less model-dependence, one can trust that they are comparatively reliable. Moreover, as widely discussed, the radiative decays of $J / \psi$ may provide an ideal place to look for the mysterious glueballs 2. Since the structure of glueballs is not clear so far, we ignore contributions from possible glueball resonances and just concentrate our focus on the direct processes which are supposed to correspond to the experimental data.

Körner et al. 3] investigated the OZI-forbidden radiative decays of orthoquarkonia in perturbative QCD and their pioneer work was done more than 20 years ago. Since then, the technique of calculating loop diagrams has been improved and the knowledge on the wavefunctions of light mesons such as $\pi, \eta, \eta^{\prime}$ is much enriched. Meanwhile the corresponding experimental measurements become more precise and make it possible to test our theoretical understanding on both the perturbative QCD calculation and the hadron-wavefunctions which are overwhelmingly governed by non-perturbative QCD.

\footnotetext{
${ }^{*}$ Supported by National Natural Science Foundation of China.
} 
In Körner et al.'s work [3], the authors tactfully dealt with the complicated Feynman integrations of four-point and five-point loop functions, i.e. the D- and E-functions [4]. They took the weak-binding approximation for both heavy and light mesons, indeed generally this approximation is reasonable and much simplifies the calculations. By the approximation, the heavy quarks $Q, \bar{Q}$ ( $c$ and $\bar{c}$ for $J / \psi$ ) and light quarks $q, \bar{q}$ in the decay product-light orthoquarkoinum are set to possess equal momenta and be on their mass shells, i.e. $p_{Q}=p_{\bar{Q}}, p_{q}=p_{\bar{q}}$ and $p_{Q}^{2}=m_{Q}^{2}, p_{q}^{2}=m_{q}^{2}$.

In the weak-binding approximation where $q$ and $\bar{q}$ have the same momentum and are on mass shell, the flavor dependence of the propagator disappears due to the on-shell condition, thus the difference of flavors does not manifest in the integration. Since $\pi^{0}$ is of structure $\frac{1}{\sqrt{2}}(d \bar{d}-u \bar{u}), \eta_{8}$ is of structure $\frac{1}{\sqrt{6}}(u \bar{u}+d \bar{d}-2 s \bar{s})$, the contributions from different flavors cancel each other and make the total width null, whereas only for $\eta_{0}=\frac{1}{\sqrt{3}}(u \bar{u}+d \bar{d}+s \bar{s})$, all contributions are added up and the result is non-zero. Therefore to get non-zero widths for $J / \psi \rightarrow \gamma \pi^{0}, \gamma \eta_{8}$, one must abandon the weak-binding approximation, namely, consider the relative momentum between $q$ and $\bar{q}$ and not let them be on mass shell.

Noting the disadvantage of this approximation, Yang [6, 7] dismissed it and re-derived relevant formulas. In his work where he neglected the mass of the light quark mass of the light meson and obtained an improved analytical expression for the rates of $J / \psi \rightarrow \eta \gamma, \eta^{\prime} \gamma$.

As $J / \psi$ is much heavier than the produced light mesons $\left(\pi^{0}, \eta, \eta^{\prime}\right)$, the 3 -momentum of the mesons is large, namely much larger than the relative momentum between $q$ and $\bar{q}$, the equal-momentum approximation as well as the approximate on-shell condition are reasonable. However, this approximation probably too simplifies the picture. Concretely, $J / \psi$ (or $\Upsilon$ ) is an $\mathrm{SU}(3)$ singlet, $\pi^{0}$ belongs to isospin- $1, \eta$ has a large fraction of $\eta_{8}$, and in $\eta^{\prime}$, the $\eta_{0}$ component dominates. Strictly, the electromagnetic interaction does not demand an isospin conservation, i.e. the isospin of photon can be either 0 or 1 . But as we take the most probable structure that isospin of photon is 0 , the $J / \psi \rightarrow \gamma \pi^{0}$ is an isospin-violating process and $J / \psi \rightarrow \gamma \eta_{8}$ is an $\mathrm{SU}(3)$ violating process and only $J / \psi \rightarrow \gamma \eta_{0}$ is an $\mathrm{SU}(3)$ conserving process. One can expect the sequence $\Gamma\left(J / \psi \rightarrow \gamma \pi^{0}\right)<\Gamma\left(J / \psi \rightarrow \gamma \eta_{8}\right)<\Gamma\left(J / \psi \rightarrow \gamma \eta_{0}\right)$ and the present experimental data on $J / \psi$ confirm this conjecture [8]. In our calculations, we will show that this sequence is completely understood in a full loop calculation, i.e. the cancellation among different quarkflavors in $\pi^{0}$ and $\eta_{8}$ causes this pattern.

We are motivated to re-evaluate the processes $J / \psi \rightarrow \gamma \pi^{0}, \gamma \eta, \gamma \eta^{\prime}$ by keeping an arbitrary relative momentum between $q$ and $\bar{q}$ and non-degenerate masses of the light quarks $(u, d, s)$. We notice that just because of this mass difference the three modes have different non-zero branching ratios. In the early work [3], the authors supposed that all processes occur via $J / \psi \rightarrow \eta_{0}+\gamma$, and the mixing of $\eta_{8}$ and $\eta_{0}$ results in the non-zero rate for $J / \psi \rightarrow \eta \gamma$.

However, as dismissing the weak-binding approximation, the advantages for simplifying the calculations are lost, namely, one cannot approximately reduce the five-point and four-point loop functions into simple three-point loop functions. It is easy to understand that under the weak-binding approximation where $q$ and $\bar{q}$ have the same momentum and are on shell, the numerators of the integrands can be properly decomposed into several groups and each of them can cancel certain factors of the denominators, so that the number of the Feynman parameters can be reduced, whereas without the approximation, generally all the terms of the 5-point and 4-point loop functions remain and the expressions cannot be further simplified (see below for details.). 
Following the standard procedure [4, we deal with the 5-point loop functions and then in term of the program [9], we evaluate integrations of the 4-point and 3-point loop functions to obtain an effective vertex [10] for $J / \psi \rightarrow \gamma g g \rightarrow \gamma+P$ where $P$ is a pseudoscalar.

Moreover, there seems to be another contribution from the tree diagram

$$
c \bar{c}(J / \psi) \rightarrow \gamma^{*} \rightarrow \gamma+q \bar{q}(P)
$$

shown in Fig.1 (a) and (b). By a simple analysis, one can immediately note that the contributions from (a) and (b) exactly cancel each other as long as the wavefunction of $\mathrm{P}$ is symmetric to the two light-quark constituents. Therefore in the radiative decays of orthoquarkonia, the leading contribution comes from the one-loop OZI forbidden processes [3, 6].

To obtain the decay amplitude, one needs to evaluate the hadronic matrix elements

$$
<P\left|V_{e f f}\right| J / \psi>
$$

where $P$ stands for $\pi^{0}, \eta$ and $\eta^{\prime}$. In the calculations, we use the light-cone wavefunction [1, 12, 13] for $P$.

In the non-relativistic model, the wavefunction of the light meson $P$ at zero-point $R_{P S}(0)$ is responsible for the hadronization effects 3, 10.

The same problem has also been investigated by some other authors [14, 15], especially an anomaly for $\Upsilon \rightarrow \gamma \eta^{\prime}$ is addressed. In their work, the gluon contents in $\eta$ and $\eta^{\prime}$ are considered and non-perturbative effects are taken into account. In the work [15], the anomaly seems to be alleviated in this approach, but the concerned assumption is still under suspicion [16. We will come back to this point again in our last section.

The paper is organized as follows. After this long introduction, in Sec.II, we present our formulation and in Sec. III, we make the numerical evaluation of the decay rates of $J / \psi \rightarrow$ $\gamma \pi^{0}, \gamma \eta, \gamma \eta^{\prime}$ and some necessary input parameters are explicitly given. To investigate the whole scenario, we further calculate the rates of $J / \psi \rightarrow \rho^{0} \gamma$ as well as $\Upsilon \rightarrow \pi^{0} \gamma, \eta \gamma, \eta^{\prime} \gamma$. We find an anomaly for $\Upsilon \rightarrow \eta^{\prime} \gamma$. The last section is devoted to a simple discussion and our conclusion. Some of the tedious details are collected in the appendices.

\section{Formulation of process $V \rightarrow P \gamma$}

\subsection{Derivation of the effective operators}

(i) For $V \rightarrow P \gamma$.

First we derive the formula for $J / \psi \rightarrow P \gamma$, where $P$ stands as a neutral pseudoscalar meson $\left(\pi^{0}, \eta\right.$ and $\left.\eta^{\prime}\right)$. As mentioned above, the tree diagrams shown in Fig.1 (a) and (b) make null contributions due to a mutual cancellation among them. The corresponding Feynman diagrams which are OZI suppressed and offer the leading contributions to the processes, are shown in Fig.2 (a) through (f).

The effective vertex for $J / \psi \rightarrow \gamma+P$ where $P$ stands for a pseudoscalar is in the form [17, 18,

$$
V_{\text {eff }}=g_{\text {eff }} \epsilon_{\alpha \beta \mu \nu} p_{V}^{\alpha} \varepsilon_{J / \psi}^{\beta} q^{\mu} \varepsilon_{\gamma}^{* \nu},
$$

where $p_{V}^{\alpha}=p_{V}^{0}=M_{J / \psi}$ is the four-momentum of $J / \psi$ in its center-of-mass frame, $\varepsilon_{J / \psi}^{\beta}$ and $\varepsilon_{\gamma}^{* \nu}$ are the polarizations of $J / \psi$ and the emitted photon, $q$ is the relative momentum of the photon 
and the pseudoscalar. The $g_{\text {eff }}$ is an effective coupling and should be derived by evaluating the corresponding Feynman diagrams and loop integrations. Concretely, this expression is at the hadron level, thus our strategy is to derive all the effective operators at the quark level by carrying out the loop integrations and then in terms of the wavefunctions of the mesons, we obtain the decay amplitude at the hadron level, indeed our goal is to derive $g_{\text {eff }}$ in eq.(2).

Without the weak-binding approximation which was adopted in literature [3, 10, and keeping the masses of the light quarks at the propagators, we re-formulate the amplitude. The amplitude of $V \rightarrow P \gamma$ can be divided into three pieces which correspond to Fig. 2 (a), (b) and (c) respectively.

For Fig. 2(a), we have

$$
\begin{aligned}
M_{A}= & \frac{i \pi^{2} e Q_{Q} g_{s}^{4} T^{a} T^{b} \otimes T^{b} T^{a}}{\left(\left(\frac{1}{2} p_{V}-q\right)^{2}-m_{Q}^{2}\right)(2 \pi)^{4}} \varepsilon^{* \mu}(\gamma) \\
& {\left[D_{1}^{\rho \nu}\left(x, m_{q}\right)\left(\frac{1}{2} p_{V}-q\right)^{\sigma} O_{\mu \sigma \rho \nu}^{\mathbf{1}}+D_{1}^{\rho \nu}\left(x, m_{q}\right) m_{Q} O_{\mu \rho \nu}^{\mathbf{2}}+D_{2}^{\nu}\left(x, m_{q}\right)\left(\frac{1}{2} p_{V}-q\right)^{\sigma} m_{Q} O_{\mu \nu \sigma}^{\mathbf{3}}\right.} \\
& \left.+D_{2}^{\nu}\left(x, m_{q}\right) m_{Q}^{2} O_{\mu \nu}^{\mathbf{4}}\right],
\end{aligned}
$$

where

$$
\begin{aligned}
& O_{\mu \sigma \rho \nu}^{\mathbf{1}}=-2 i g_{\rho \nu} \varepsilon_{\beta \sigma \mu a} \bar{v}_{Q} \gamma^{a} u_{Q} \bar{u}_{q} \gamma^{\beta} \gamma_{5} v_{q}+2 i \varepsilon_{\nu \sigma \mu \theta} \bar{v}_{Q} \gamma^{\theta} u_{Q} \bar{u}_{q} \gamma_{\rho} \gamma_{5} v_{q}, \\
& O_{\mu \rho \nu}^{2}=-2 g_{\alpha \mu} g_{a \nu} \varepsilon^{\beta a \alpha b} \bar{v}_{Q} \sigma_{\rho \beta} u_{Q} \bar{u}_{q} \gamma_{b} \gamma_{5} v_{q}+g_{\rho \mu} g_{a \nu} \varepsilon^{\beta a \alpha b} \bar{v}_{Q} \sigma_{\rho \beta} u_{Q} \bar{u}_{q} \gamma_{b} \gamma_{5} v_{q}, \\
& O_{\mu \nu \sigma}^{\mathbf{3}}=-2 g_{\alpha \sigma} g_{a \nu} \varepsilon^{\beta a \alpha b} \bar{v}_{Q} \sigma_{\beta \mu} u_{Q} \bar{u}_{q} \gamma_{b} \gamma_{5} v_{q}-2 g_{\beta \mu} g_{a \nu} \varepsilon^{\beta a \alpha b} \bar{v}_{Q} \sigma_{\alpha \sigma} u_{Q} \bar{u}_{q} \gamma_{b} \gamma_{5} v_{q} \\
& +g_{\sigma \mu} g_{a \nu} \varepsilon^{\beta a \alpha b} \bar{v}_{Q} \sigma_{\alpha \beta} u_{Q} \bar{u}_{q} \gamma_{b} \gamma_{5} v_{q}, \\
& O_{\mu \nu}^{4}=-2 i g_{\beta \mu} g_{a \nu} \varepsilon^{\beta a \alpha b} \bar{v}_{Q} \gamma_{\alpha} u_{Q} \bar{u}_{q} \gamma_{b} \gamma_{5} v_{q} .
\end{aligned}
$$

The functions $D_{1}^{\rho \nu}\left(x, m_{q}\right), D_{2}^{\nu}\left(x, m_{q}\right)$ are integrals of four-point functions over the internal momentum $k$ whose explicit forms are given in appendix A.

For Fig.2 (b), the amplitude reads

$$
\begin{aligned}
M_{B}= & \frac{i \pi^{2} e Q_{Q} g_{s}^{4} T^{a} T^{b} \otimes T^{b} T^{a}}{\left[\left(\frac{1}{2} p_{V}-q\right)^{2}-m_{Q}^{2}\right](2 \pi)^{4}} \varepsilon^{* \mu}(\gamma) \\
& {\left[D_{1}^{\rho \nu}\left(x, m_{q}\right)\left(\frac{1}{2} p_{V}-q\right)^{\sigma} O_{\mu \sigma \rho \nu}^{\mathbf{1}}-D_{1}^{\rho \nu}\left(x, m_{q}\right) m_{Q} O_{\mu \rho \nu}^{\mathbf{2}}-D_{2}^{\nu}\left(x, m_{q}\right)\left(\frac{1}{2} p_{V}-q\right)^{\sigma} m_{Q} O_{\mu \nu \sigma}^{\mathbf{3}}\right.} \\
& \left.+D_{2}^{\nu}\left(x, m_{q}\right) m_{Q}^{2} O_{\mu \nu}^{\mathbf{4}}\right],
\end{aligned}
$$

and the amplitude corresponding to Fig.2 (c) is

$M_{C}=\frac{e Q_{Q} g_{s}^{4} T^{a} T^{b} \otimes T^{b} T^{a} i \pi^{2}}{(2 \pi)^{4}} \varepsilon^{* \mu}(\gamma)\left[E_{1}^{\nu \rho \sigma}\left(x, m_{q}\right) O_{\mu \nu \rho \sigma}^{\mathbf{5}}+E_{2}^{\nu \sigma}\left(x, m_{q}\right) m_{Q} O_{\mu \nu \sigma}^{\mathbf{6}}+E_{3}^{\rho \sigma}\left(x, m_{q}\right) m_{Q} O_{\mu \rho \sigma}^{\mathbf{7}}\right]$,

where

$$
\begin{aligned}
& O_{\mu \nu \rho \sigma}^{\mathbf{5}}=i \varepsilon_{\nu \mu \rho \sigma} \bar{v}_{Q} \gamma_{\beta} u_{Q} \bar{u}_{q} \gamma^{\beta} \gamma_{5} v_{q}-2 i \varepsilon_{\nu \mu \rho \theta} \bar{v}_{Q} \gamma_{\sigma} u_{Q} \bar{u}_{q} \gamma^{\theta} \gamma_{5} v_{q}, \\
& O_{\mu \nu \sigma}^{\mathbf{6}}=g_{c \sigma} g_{\mu \nu} \varepsilon^{\beta c \alpha \theta} \bar{v}_{Q} \sigma_{\alpha \beta} u_{Q} \bar{u}_{q} \gamma_{\theta} \gamma_{5} v_{q}, \\
& O_{\mu \rho \sigma}^{\mathbf{7}}=g_{c \sigma} g_{\mu \rho} \varepsilon^{\beta c \alpha \theta} \bar{v}_{Q} \sigma_{\alpha \beta} u_{Q} \bar{u}_{q} \gamma_{\theta} \gamma_{5} v_{q} .
\end{aligned}
$$


The functions $E_{1}^{\nu \rho \sigma}\left(x, m_{q}\right), E_{2}^{\nu \sigma}\left(x, m_{q}\right), E_{3}^{\rho \sigma}\left(x, m_{q}\right)$ are three independent integrals of fivepoint functions over the internal momentum $k$ and their explicit expressions are also collected in appendix A.

The contributions of the other three diagrams Fig. 2 (d,e,f) are similar to that of the first three $(a, b, c)$, to save space, we omit their expressions in the context.

It is noted that the contribution of Fig.2 (c) is a five-point Green's function, namely there are five propagators in the loop. As long as the weak-binding approximation is adopted, the fivepoint functions $E_{1}^{\nu \rho \sigma}\left(x, m_{q}\right), E_{2}^{\nu \sigma}\left(x, m_{q}\right), E_{3}^{\rho \sigma}\left(x, m_{q}\right)$ can be decomposed into sums of two-point and three-point functions, so that the calculations are much simplified and analytical expressions are eventually derived [3, 6]. By contrary, without the weak-binding approximation, such decomposition is impossible, unfortunately. Following Denner and Dittmaier [4, we decompose the five-point functions into a sum of several four-point functions which cannot be integrated out analytically. Instead, we will calculate the integrals numerically. Some details about the integration are presented in Appendix B.

(ii) For $V \rightarrow \rho^{0} \gamma$.

Serving as a check, we also calculate the branching ratio of $J / \psi \rightarrow \rho^{0} \gamma$ in comparison with that of $J / \psi \rightarrow \pi^{0} \gamma$. The Feynman diagrams are the same as that for $J / \psi \rightarrow \pi^{0} \gamma$, and the explicit expressions of the amplitudes are given in Appendix A for saving space.

\subsection{The hadronic matrix elements}

With the Feynman diagrams of Fig.2 we derive an effective lagrangian at the quark level. To obtain the hadronic matrix elements and then finally the decay rates, one has to evaluate the hadronic matrix elements. It is well known that the hadronization happens at the energy scale of $\Lambda_{Q C D}$ which is in the range of non-perturbative QCD, so far, there is no any reliable way to evaluate the hadronic matrix elements. To do the job, we need to invoke concrete models. Since the produced meson is relatively light and its three-momentum is larger than $\Lambda_{Q C D}$, the light-cone wavefunctions seem to be plausible for description of the light mesons [13, 19, 20].

The matrix elements are

$$
\begin{aligned}
& \left\langle P\left|\bar{q} \gamma_{\alpha} \gamma_{5} q \sum_{i}\left(C_{i}\left(x, m_{q}\right) O_{i}\right) \bar{Q} \gamma_{\beta} Q\right| V\right\rangle \\
& =-i f_{P} p_{P} \alpha \int_{0}^{1} d x \phi_{P}(\mu, x) \sum_{i}\left(C_{i}\left(x, m_{q}\right) O_{i}\right) i f_{V} \varepsilon_{V} M_{V}, \\
& \left\langle P\left|\bar{q} \gamma_{\alpha} \gamma_{5} q \sum_{i}\left(C_{i}\left(x, m_{q}\right) O_{i}\right) \bar{Q} \sigma_{\beta \rho} Q\right| V\right\rangle \\
& =-i f_{P} p_{P \alpha} \int_{0}^{1} d x \phi_{P}(\mu, x) \sum_{i}\left(C_{i}\left(x, m_{q}\right) O_{i}\right) i f_{V} i\left(\varepsilon_{V} p_{V} \rho-p_{V} \varepsilon_{V} \rho\right),
\end{aligned}
$$

where $O_{i}$ 's and $C_{i}$ 's are the operators and their coefficients derived in last sub-section. For the pseudoscalars, the $\mathrm{SU}(3)$ flavor wavefuctions are

$$
\pi^{0}=\frac{u \bar{u}-d \bar{d}}{\sqrt{2}}, \eta_{0}=\frac{d \bar{d}+u \bar{u}+s \bar{s}}{\sqrt{3}} \text { and } \eta_{8}=\frac{d \bar{d}+u \bar{u}-2 s \bar{s}}{\sqrt{6}}
$$


$\eta$ and $\eta^{\prime}$ are mixutures of $\eta_{0}$ and $\eta_{8}$

$$
\eta=\cos \theta \eta_{8}-\sin \theta \eta_{0}, \quad \eta^{\prime}=\sin \theta \eta_{8}+\cos \theta \eta_{0} .
$$

The normalization of the light-cone wavefunction is defined as

$$
\int_{0}^{1} d x \phi(\mu, x)=1
$$

The explicit forms of the light-cone wavefunctions of the light mesons can be different. In our later calculations, we take three different types of wavefunctions which are given in literatures 11, 12, 13, 21] as

$$
\begin{aligned}
\phi_{1}(\mu, x) & =6 x(1-x) \\
\phi_{2}(\mu, x) & =30 x^{2}(1-x)^{2} \\
\phi_{3}(\mu, x) & =\frac{15}{2}(1-2 x)^{2}\left[1-(1-2 x)^{2}\right] .
\end{aligned}
$$

Finally we obtain the hadronic matrix elements of $M_{A}, M_{B}$ and $M_{C}$ as following

$$
\begin{aligned}
& \left\langle P \gamma\left|M_{A}\right| V\right\rangle=i \pi^{2} e Q_{Q} g_{s}^{4} T^{a} T^{b} \otimes T^{b} T^{a} \frac{1}{(2 \pi)^{4}} \frac{1}{\left(\frac{1}{2} p_{V}-q\right)^{2}-m_{Q}^{2}} \frac{1}{N_{c}^{2}} \varepsilon^{* \mu}(\gamma) \int_{0}^{1} d x \phi_{P}(\mu, x) \\
& \left\{f_{P} f_{V} M_{V}\left[-2 i g_{\rho \nu} \epsilon_{\beta \sigma \mu a} p_{P}^{\beta} \varepsilon_{V}^{a}+2 i \epsilon_{\nu \sigma \mu \theta} p_{P} \varepsilon_{V}^{\theta}\right] \sum_{q=u, d, s}\left(D_{1}^{\rho \nu}\left(x, m_{q}\right)\right)\left(\frac{1}{2} p_{V}-q\right)^{\sigma}\right. \\
& +i f_{P} f_{V}\left[-2 g_{\alpha \mu} g_{a \nu} \epsilon^{\beta a \alpha b} p_{P}\left(\varepsilon_{V} p_{V} \beta-\varepsilon_{V \beta} p_{V} \rho\right)\right. \\
& \left.+g_{\rho \mu} g_{a \nu} \epsilon^{\beta a \alpha b} p_{P}\left(\varepsilon_{V} \alpha p_{V} \beta-\varepsilon_{V} \beta p_{V} \alpha\right)\right] \sum_{q=u, d, s}\left(D_{1}^{\rho \nu}\left(x, m_{q}\right)\right) m_{Q} \\
& +i f_{P} f_{V}\left[-2 g_{\alpha \sigma} g_{a \nu} \epsilon^{\beta a \alpha b} p_{P} b\left(\varepsilon_{V} \beta p_{V} \mu-\varepsilon_{V \mu} p_{V} \beta\right)\right. \\
& -2 g_{\beta \mu} g_{a \nu} \epsilon^{\beta a \alpha b} p_{P b}\left(\varepsilon_{V} \alpha p_{V} \sigma-\varepsilon_{V \sigma} p_{V \alpha}\right) \\
& \left.+g_{\sigma \mu} g_{a \nu} \epsilon^{\beta a \alpha b} p_{P b}\left(\varepsilon_{V} \alpha p_{V} \beta-\varepsilon_{V} \beta p_{V} \alpha\right)\right] \sum_{q=u, d, s}\left(D_{2}^{\nu}\left(x, m_{q}\right)\right)\left(\frac{1}{2} p_{V}-q\right)^{\sigma} m_{Q} \\
& \left.+f_{P} f_{V} M_{V}\left[-2 i g_{\beta \mu} g_{a \nu} \epsilon^{\beta a \alpha b} p_{P b} \varepsilon_{V} \alpha\right] \sum_{q=u, d, s}\left(D_{2}^{\nu}\left(x, m_{q}\right)\right) m_{Q}^{2}\right\}, \\
& \left\langle P \gamma\left|M_{B}\right| V\right\rangle=i \pi^{2} e Q_{Q} g_{s}^{4} T^{a} T^{b} \otimes T^{b} T^{a} \frac{1}{(2 \pi)^{4}} \frac{1}{\left(\frac{1}{2} p_{V}-q\right)^{2}-m_{Q}^{2}} \frac{1}{N_{c}^{2}} \varepsilon^{* \mu}(\gamma) \int_{0}^{1} d x \phi_{P}(\mu, x) \\
& \left\{f_{P} f_{V} M_{V}\left[-2 i g_{\rho \nu} \epsilon_{\beta \sigma \mu a} p_{P}^{\beta} \varepsilon_{V}^{a}+2 i \epsilon_{\nu \sigma \mu \theta} p_{P} \varepsilon_{V}^{\theta}\right] \sum_{q=u, d, s}\left(D_{1}^{\rho \nu}\left(x, m_{q}\right)\right)\left(\frac{1}{2} p_{V}-q\right)^{\sigma}\right. \\
& -i f_{P} f_{V}\left[-2 g_{\alpha \mu} g_{a \nu} \epsilon^{\beta a \alpha b} p_{P b}\left(\varepsilon_{V} p_{V} \beta-\varepsilon_{V \beta} p_{V} \rho\right)\right. \\
& \left.+g_{\rho \mu} g_{a \nu} \epsilon^{\beta a \alpha b} p_{P}\left(\varepsilon_{V \alpha} p_{V} \beta-\varepsilon_{V} \beta p_{V} \alpha\right)\right] \sum_{q=u, d, s}\left(D_{1}^{\rho \nu}\left(x, m_{q}\right)\right) m_{Q} \\
& -i f_{P} f_{V}\left[-2 g_{\alpha \sigma} g_{a \nu} \epsilon^{\beta a \alpha b} p_{P}\left(\varepsilon_{V} \beta p_{V} \mu-\varepsilon_{V} p_{V} \beta\right)\right. \\
& -2 g_{\beta \mu} g_{a \nu} \epsilon^{\beta a \alpha b} p_{P b}\left(\varepsilon_{V} \alpha p_{V} \sigma-\varepsilon_{V} p_{V} \alpha\right)
\end{aligned}
$$




$$
\begin{aligned}
& \left.+g_{\sigma \mu} g_{a \nu} \epsilon^{\beta a \alpha b} p_{P b}\left(\varepsilon_{V} \alpha p_{V} \beta-\varepsilon_{V} p_{V} \alpha\right)\right] \sum_{q=u, d, s}\left(D_{2}^{\nu}\left(x, m_{q}\right)\right)\left(\frac{1}{2} p_{V}-q\right)^{\sigma} m_{Q} \\
& \left.+f_{P} f_{V} M_{V}\left[-2 i g_{\beta \mu} g_{a \nu} \epsilon^{\beta a \alpha b} p_{P b} \varepsilon_{V} \alpha\right] \sum_{q=u, d, s}\left(D_{2}^{\nu}\left(x, m_{q}\right)\right) m_{Q}^{2}\right\},
\end{aligned}
$$

and

$$
\begin{aligned}
& \left\langle P \gamma\left|M_{C}\right| V\right\rangle=i \pi^{2} e Q_{Q} g_{s}^{4} T^{a} T^{b} \otimes T^{b} T^{a} \frac{1}{(2 \pi)^{4}} \frac{1}{N_{c}^{2}} \varepsilon^{* \mu}(\gamma) \int_{0}^{1} d x \phi_{P}(\mu, x) \\
& \left\{f_{P} f_{V} M_{V}\left[i \epsilon_{\nu \mu \rho \sigma} p_{P}^{\beta} \varepsilon_{V} \beta-2 i \epsilon_{\nu \mu \rho \theta} p_{P}^{\theta} \varepsilon_{V}\right] \sum_{q=u, d, s}\left(E_{1}^{\nu \rho \sigma}\left(x, m_{q}\right)\right)\right. \\
& +i f_{P} f_{V}\left[g_{\sigma c} \epsilon^{\beta c \tau \theta} g_{\mu \nu} p_{P} \theta\left(\varepsilon_{V} p_{V} \beta-\varepsilon_{V} \beta p_{V}\right)\right] \sum_{q=u, d, s}\left(E_{2}^{\nu \sigma}\left(x, m_{q}\right)\right) m_{Q} \\
& \left.+i f_{P} f_{V}\left[g_{\sigma c} \epsilon^{\beta c \tau \theta} g_{\mu \rho} p_{P} \theta\left(\varepsilon_{V} p_{V} \beta-\varepsilon_{V} \beta p_{V}\right)\right] \sum_{q=u, d, s}\left(E_{3}^{\rho \sigma}\left(x, m_{q}\right)\right) m_{Q}\right\} .
\end{aligned}
$$

\section{$3 \quad$ Numerical results}

In this section, we present our numerical results.

In the numerical computations, there is a mild Infrared (IR) divergence problem. Namely, when we carry out the loop integration and convolution integrals of the effective operators with the light-cone wavefunction of the produced meson, an IR-divergence emerges, but it is not as serious as that in the B-meson decays and can be removed in simple ways. Our strategy to deal with the IR problem is standard, namely we assign a small mass to the gluon and vary it to check if the result is stable. Practically, we set the small mass to be from $10^{-4} \mathrm{MeV}$ to $10^{-6} \mathrm{MeV}$ and find that the result has only negligible changes. Therefore we can trust the obtained result which is free of the IR problem. Our final results given in all the following tables correspond to the gluon mass of $10^{-5} \mathrm{MeV}$.

\subsection{Input parameters}

The input parameters which we are going to use in the numerical computations are taken as follows [8, 10, 19, 22, 23]: $f_{J / \psi}=551 \mathrm{MeV}, f_{\Upsilon}=710 \mathrm{MeV}, f_{\pi}=131 \mathrm{MeV}, f_{\eta}=f_{\eta^{\prime}}=$ $157 \mathrm{MeV}, f_{\rho}=198 \mathrm{MeV}, M_{J / \psi}=3096.87 \mathrm{MeV}, M_{\Upsilon}=9460.3 \mathrm{MeV}, m_{\pi}=134.9766 \mathrm{MeV}$, $m_{\eta}=547.75 \mathrm{MeV}, m_{\eta^{\prime}}=957.78 \mathrm{MeV}, m_{\rho}=775.8 \mathrm{MeV}, \alpha_{s}\left(m_{c}\right)=0.26, \alpha_{s}\left(m_{b}\right)=0.17$, $m_{c}=1300 \mathrm{MeV}, m_{b}=4700 \mathrm{MeV}$, the mixing angle $\theta=-11^{\circ}$, and three possible distribution amplitudes of pseudoscalar meson are given in Eqs. (81910).

We will present the the resultant decay rates corresponding to the three different distribution amplitudes respectively in the following tables.

\subsection{Numerical results of process $V \rightarrow P \gamma$}

With the above parameters, the theoretical values for the decay width of these processes in the rest frame of $J / \psi$, are shown in Table 1 . 
Table 1: The decay branching ratio of $J / \psi \rightarrow \pi^{0} \gamma, J / \psi \rightarrow \eta \gamma$ and $J / \psi \rightarrow \eta^{\prime} \gamma$ in the rest frame of $J / \psi$ and the three columns correspond to the three different parton distribution amplitudes of the produced pseudoscalar mesons $\left(\pi^{0}, \eta, \eta^{\prime}\right)$.

\begin{tabular}{|c|c|c|c|c|c|c|c|}
\hline Processes & $m_{u}$ & $m_{d}$ & $m_{s}$ & $10^{5} \times B R\left(\phi_{1}\right)$ & $10^{5} \times B R\left(\phi_{2}\right)$ & $10^{5} \times B R\left(\phi_{3}\right)$ & Experimental data \\
\hline \multirow{5}{*}{$J / \psi \rightarrow \pi^{0} \gamma$} & 1.5 & 4 & 0 & 2.71325 & 0.402025 & 25.9819 & \multirow{5}{*}{$(3.9 \pm 1.3) \times 10^{-5}$} \\
\hline & 2 & 4 & 0 & 0.895075 & 0.292837 & 9.09646 & \\
\hline & 3 & 5 & 0 & 1.27478 & 0.428238 & 11.8012 & \\
\hline & 3 & 7 & 0 & 6.43086 & 2.21318 & 55.5486 & \\
\hline & 4 & 6 & 0 & 1.64827 & 0.565255 & 14.1993 & \\
\hline \multirow{7}{*}{$J / \psi \rightarrow \eta \gamma$} & $m_{u}$ & $m_{d}$ & $m_{s}$ & $10^{4} \times B R\left(\phi_{1}\right)$ & $10^{4} \times B R\left(\phi_{2}\right)$ & $10^{4} \times B R\left(\phi_{3}\right)$ & \multirow{7}{*}{$(8.6 \pm 0.8) \times 10^{-4}$} \\
\hline & 2 & 4 & 80 & 2.41823 & 1.68707 & 7.23647 & \\
\hline & 2 & 5 & 90 & 2.46188 & 1.69669 & 7.44466 & \\
\hline & 3 & 5 & 100 & 2.56809 & 1.72051 & 8.12141 & \\
\hline & 2 & 6 & 110 & 2.48921 & 1.6743 & 7.97546 & \\
\hline & 4 & 6 & 120 & 2.50536 & 1.66464 & 8.12365 & \\
\hline & 3 & 7 & 130 & 2.55453 & 1.65799 & 8.6264 & \\
\hline \multirow{7}{*}{$J / \psi \rightarrow \eta^{\prime} \gamma$} & $m_{u}$ & $m_{d}$ & $m_{s}$ & $10^{3} \times B R\left(\phi_{1}\right)$ & $10^{3} \times B R\left(\phi_{2}\right)$ & $10^{3} \times B R\left(\phi_{3}\right)$ & \multirow{7}{*}{$(4.3 \pm 0.3) \times 10^{-3}$} \\
\hline & 2 & 4 & 80 & 1.19297 & 1.07802 & 1.92765 & \\
\hline & 2 & 5 & 90 & 1.18068 & 1.07066 & 1.90548 & \\
\hline & 3 & 5 & 100 & 1.17033 & 1.06154 & 1.87308 & \\
\hline & 2 & 6 & 110 & 1.15948 & 1.05165 & 1.84638 & \\
\hline & 4 & 6 & 120 & 1.14416 & 1.03956 & 1.8022 & \\
\hline & 3 & 7 & 130 & 1.12954 & 1.02747 & 1.76655 & \\
\hline
\end{tabular}

Obviously, the same procedure can be applied to the radiative decays of $\Upsilon \rightarrow \pi^{0}\left(\eta, \eta^{\prime}\right) \gamma$. We calculate the widths with the same parameters by replacing c-quark in $J / \psi$ by b-quark in $\Upsilon$, $Q_{c}$ by $Q_{b}$ and $m_{c}$ by $m_{b}$. Then we obtain the numerical results which are shown in Table 2 . 
Table 2: The decay branching ratio of $\Upsilon \rightarrow \pi^{0} \gamma, \Upsilon \rightarrow \eta \gamma$ and $\Upsilon \rightarrow \eta^{\prime} \gamma$ in the rest frame of $\Upsilon$ and the three columns correspond to the three different distribution amplitudes of the produced pseudoscalar mesons.

\begin{tabular}{|c|c|c|c|c|c|c|c|}
\hline Processes & $m_{u}$ & $m_{d}$ & $m_{s}$ & $10^{5} \times B R\left(\phi_{1}\right)$ & $10^{5} \times B R\left(\phi_{2}\right)$ & $10^{5} \times B R\left(\phi_{3}\right)$ & Experimental data \\
\hline \multirow{5}{*}{$\Upsilon \rightarrow \pi^{0} \gamma$} & 1.5 & 4 & 0 & 0.958428 & 0.674172 & 2.78981 & \multirow{5}{*}{$<3 \times 10^{-5}$} \\
\hline & 2 & 4 & 0 & 0.634743 & 0.478474 & 1.60618 & \\
\hline & 3 & 5 & 0 & 0.575449 & 0.618915 & 0.445925 & \\
\hline & 3 & 7 & 0 & 1.92777 & 2.81325 & 0.5723 & \\
\hline & 4 & 6 & 0 & 0.4889 & 0.723972 & 0.071549 & \\
\hline \multirow{7}{*}{$\Upsilon \rightarrow \eta \gamma$} & $m_{u}$ & $m_{d}$ & $m_{s}$ & $10^{5} \times B R\left(\phi_{1}\right)$ & $10^{5} \times B R\left(\phi_{2}\right)$ & $10^{5} \times B R\left(\phi_{3}\right)$ & \multirow{7}{*}{$<2.1 \times 10^{-5}$} \\
\hline & 2 & 4 & 80 & 0.264287 & 0.037964 & 9.31202 & \\
\hline & 2 & 5 & 90 & 0.467742 & 0.0426104 & 13.9632 & \\
\hline & 3 & 5 & 100 & 0.772832 & 0.0571983 & 19.97 & \\
\hline & 2 & 6 & 110 & 1.20528 & 0.0916147 & 27.4375 & \\
\hline & 4 & 6 & 120 & 1.80313 & 0.158933 & 36.6147 & \\
\hline & 3 & 7 & 130 & 2.59821 & 0.276932 & 47.4917 & \\
\hline \multirow{7}{*}{$\Upsilon \rightarrow \eta^{\prime} \gamma$} & $m_{u}$ & $m_{d}$ & $m_{s}$ & $10^{4} \times B R\left(\phi_{1}\right)$ & $10^{4} \times B R\left(\phi_{2}\right)$ & $10^{4} \times B R\left(\phi_{3}\right)$ & \multirow{7}{*}{$<1.6 \times 10^{-5}$} \\
\hline & 2 & 4 & 80 & 9.58491 & 8.54894 & 14.7284 & \\
\hline & 2 & 5 & 90 & 9.57026 & 8.55094 & 14.6 & \\
\hline & 3 & 5 & 100 & 9.54632 & 8.5466 & 14.4558 & \\
\hline & 2 & 6 & 110 & 9.51302 & 7.99717 & 14.2925 & \\
\hline & 4 & 6 & 120 & 9.47596 & 8.51945 & 14.1333 & \\
\hline & 3 & 7 & 130 & 9.42768 & 8.49574 & 13.9498 & \\
\hline
\end{tabular}

As discussed above, as a check, we evaluate the decay width of $J / \psi \rightarrow \rho^{0} \gamma$ which corresponds to an effective three-vector vertex and has not been observed yet. The results are tabulated in Table 3.

Table 3: The decay branching ratio of $J / \psi \rightarrow \rho^{0} \gamma$ in the rest frame of $J / \psi$, and the three columns correspond to the three different distribution amplitudes of $\rho^{0}$.

\begin{tabular}{|c|c|c|c|c|c|c|c|}
\hline Processes & $m_{u}$ & $m_{d}$ & $m_{s}$ & $10^{13} \times B R\left(\phi_{1}\right)$ & $10^{13} \times B R\left(\phi_{2}\right)$ & $10^{13} \times B R\left(\phi_{3}\right)$ & Experimental data \\
\hline$J / \psi \rightarrow \rho^{0} \gamma$ & 4 & 6 & 0 & 4.74477 & 2.07037 & 37.9532 & - \\
\hline
\end{tabular}




\section{Conclusion and Discussion}

In this work, we re-study the OZI forbidden radiative decays of othoquarkonia in the framework of perturbative QCD. In the process, we do not take the weak-binding approximation or set the light quark mass to be zero and carry out a complete integration of the five- and four-point functions. In this scenario, we can take into account the $\mathrm{SU}(3)$ and isospin violation which would result in non-zero rates for $J / \psi \rightarrow \pi^{0} \gamma$ and $J / \psi \rightarrow \eta_{8} \gamma$. However, on the other side, without such approximation, we cannot derive elegant analytical expressions for the amplitudes as done by Körner et al. 3] and Yang [6]. Instead, we need to invoke complicated computer programs and we follow the recipe given by Denner and Dittmaier [4 to reduce the five-point functions into a sum of four-point functions and then use the developed computer program "LoopTools" 9] to carry out the integrations.

Another difficult point is to evaluate the hadronic matrix elements which are fully governed by the non-perturbative QCD. The initial heavy quarkonium $(J / \psi$ or $\Upsilon)$ are composed of only heavy quarks, so that the on-shell approximation is reasonable and its contribution to the amplitude can be described by its wavefunction at origin, i.e. $R_{V}(0)[3,10$. As we abandon the weak-binding approximation for the produced light meson, the simple description in [3] for the produced meson by $R_{P S}(0)$ [3, 10] can no longer be adopted. Since the produced pseudoscalar meson is light, the light-cone wavefunctions seem to be applicable for the calculations. There are several typical different light-cone distribution amplitudes for the light mesons, so far, one cannot determine which one is the most suitable. Thus we adopt all the three for our calculations and the corresponding results are listed in the tables of last section.

Our results for $J / \psi \rightarrow P \gamma$ where $P$ stands for $\pi^{0}, \eta, \eta^{\prime}$, are quantitatively in agreement with the experimental measurements. For $J / \psi \rightarrow \pi^{0} \gamma$ which is an isospin violating process because it is proportional to an asymmetry of $\mathrm{u}$ and $\mathrm{d}$ quarks, within reasonable ranges of the masses of $\mathrm{u}$ and $\mathrm{d}$ quarks, all the three distribution amplitudes can result in values in agreement with data. For $J / \psi \rightarrow \eta \gamma$, it seems that only $\phi_{3}$ can give the values in good agreement with data. For $J / \psi \rightarrow \eta^{\prime} \gamma$, all the obtained values are slightly smaller than the data. This small declination can be understood, because theoretically the results depend on the masses of light quarks and the QCD coupling, (here we do not include the running of $\alpha_{s}$ as in [3]), and experimentally, all the concerned widths are small and certain measurement errors are unavoidable. We can expect that the CLEO and BES III which will be running in 2007, can make more precise measurements to testify the results. Moreover, we calculate the decay width of $J / \psi \rightarrow \rho^{0} \gamma$ which has not been measured yet. This result will be measured in near future and the data can provide some information about our understanding of both perturbative and non-perturbative QCD (the ansatz for light-cone wavefunctions of mesons).

As we turn to the radiative decays of $\Upsilon$, the situation seems peculiar. It is noted that $\Upsilon \rightarrow \pi^{0} \gamma$ is related to isospin violation, $\Upsilon \rightarrow \eta \gamma$ is related to $\mathrm{SU}(3)$ violation because $\eta$ has a large fraction of $\eta_{8}$, only $\Upsilon \rightarrow \eta^{\prime} \gamma$ conserves $\mathrm{SU}(3)$, as $\eta^{\prime}$ contains mainly $\eta_{0}$. Thus one can expect $\Gamma\left(\Upsilon \rightarrow \pi^{0} \gamma\right)<\Gamma(\Upsilon \rightarrow \eta \gamma)<\Gamma\left(\Upsilon \rightarrow \eta^{\prime} \gamma\right)$. For radiative decays of $J / \psi$, this sequence obviously holds and our calculations confirm this pattern. However, for $\Upsilon$, the measurements seem not to follow the pattern [8]. Of course the data only set upper bounds on these decay modes, there is still some possibility to upset this pattern.

Our theoretical results for $\Upsilon$ still follow the sequence, and for $\Upsilon \rightarrow \pi^{0} \gamma$ and $\Upsilon \rightarrow \eta \gamma$, the values are consistent with the experimental upper bonds, but for $\Upsilon \rightarrow \eta^{\prime} \gamma$, the calculated value is obviously larger than the upper bound set by the present experimental measurement. It may 
indicate some anomaly in these decay modes of $\Upsilon$ and the reason is worth further studies both theoretically and experimentally.

The anomaly problem has been observed by several authors [6, 14, 15, 16], all agree that in the perturbative framework which is the main content of this work, this anomaly exists. To reconcile the theoretical results with the data, the gluon contents in $\eta$ and $\eta^{\prime}$ are considered 14, 15, 16], and the non-perturbative matrix elements $<0|G \tilde{G}| \eta^{\left({ }^{\prime}\right)}>$ was evaluated by Feldman and Kroll [14. It seems that the non-perturbative effects alleviate the discrepancy between the theoretical results and data. However, as pointed in [16], the problem is not fully solved yet and needs to be investigated further. In our work, we are only dealing with the perturbative part and confirm existence of the anomaly as discovered before. We will investigate the anomaly and the probable non-perturbative effects in a wider range. Moreover, in the works [6, 14, 15, 16, the $\gamma \pi^{0}$ case was dismissed because of its smallness, by contraries, we explicitly keep the light-quark masses and obtain the corresponding results for $\gamma \pi^{0}$ final state in the radiative decays of both $J / \psi$ and $\Upsilon$.

Our starting point is indeed the same as that of Körner et al. [3] and Yang [6], except we do not take any approximation and carry out the full integration of the five- and four-point functions. We find that the numerical results for $J / \psi$ are consistent with data, but there is an anomaly for $\Upsilon \rightarrow \eta^{\prime} \gamma$ if the present measurement is correct. All these need more and careful investigations.

Acknowledgement:

This work is partly supported by the National Natural Science Foundation of China. We benefit greatly from discussions with Dr. Y.D. Yang and he kindly introduced his work on this subject to us. We are very grateful to J.P. Ma for his encouragement and fruitful discussions. Appendix A

The integrations corresponding to Fig.2 (a), (b) and (c) are

$$
\begin{aligned}
M_{A} \quad & =\int \frac{d^{4} k}{(2 \pi)^{4}} \varepsilon^{* \mu}(\gamma) \overline{v_{Q}}\left(p_{2}\right)\left(-i g_{s} T^{a} \gamma_{\alpha}\right) \frac{i}{p_{1}-\not 1+\not k-m_{Q}}\left(-i g_{s} T^{b} \gamma_{\beta}\right) \frac{i}{\not p_{1}-\not 1-m_{Q}} \\
& \left(-i e Q_{Q} \gamma_{\mu}\right) u_{Q}\left(p_{1}\right) \overline{u_{q}}\left(p_{3}\right)\left(-i g_{s} T^{b} \gamma^{\beta}\right) \frac{i}{\not p_{3}+\not k-m_{q}}\left(-i g_{s} T^{a} \gamma^{\alpha}\right) v_{q}\left(p_{4}\right) \\
& \frac{-i}{\left(p_{1}+p_{2}+k-q\right)^{2}} \frac{-i}{k^{2}} \\
& =\int \frac{d^{4} k}{(2 \pi)^{4}} e Q_{Q} g_{s}^{4} T^{a} T^{b} \otimes T^{b} T^{a} \bar{v}_{Q}\left(p_{2}\right) \gamma_{\alpha}\left(\not p_{1}-\not q+\not k+m_{Q}\right) \gamma_{\beta}\left(\not p_{1}-\not q+m_{Q}\right) \gamma_{\mu} u_{Q}\left(p_{1}\right) \\
& \overline{u_{q}}\left(p_{3}\right) \gamma^{\beta}\left(\not p_{3}+\not k+m_{q}\right) \gamma^{\alpha} v_{q}\left(p_{4}\right) \varepsilon^{* \mu}(\gamma) \\
& \frac{1}{k^{2}\left(p_{1}+p_{2}+k-q\right)^{2}\left[\left(p_{1}-q+k\right)^{2}-m_{Q}^{2}\right]\left[\left(p_{1}-q\right)^{2}-m_{Q}^{2}\right]\left[\left(p_{3}+k\right)^{2}-m_{q}^{2}\right]} \\
& =\int \frac{d^{4} k}{(2 \pi)^{4}} \varepsilon^{* \mu}(\gamma) \bar{v}_{Q}\left(p_{2}\right)\left(-i e Q_{Q} \gamma_{\mu}\right) \frac{i}{\not 1-\not p_{2}-m_{Q}}\left(-i g_{s} T^{a} \gamma_{\alpha}\right) \frac{i}{\not p_{1}+\not k-m_{Q}} \\
M_{B} & \left(-i g_{s} T^{b} \gamma_{\beta}\right) u_{Q}\left(p_{1}\right) \bar{u}_{q}\left(p_{3}\right)\left(-i g_{s} T^{b} \gamma^{\beta}\right) \frac{i}{\not p_{3}+\not k-m_{q}}\left(-i g_{s} T^{a} \gamma^{\alpha}\right) v_{q}\left(p_{4}\right)
\end{aligned}
$$




$$
\begin{aligned}
& \frac{-i}{\left(p_{1}+p_{2}+k-q\right)^{2}} \frac{-i}{k^{2}} \\
& =\int \frac{d^{4} k}{(2 \pi)^{4}} \varepsilon^{* \mu}(\gamma) e Q_{c} g_{s}^{4} T^{a} T^{b} \otimes T^{b} T^{a} \bar{v}_{Q}\left(p_{2}\right) \gamma_{\mu}\left(\not q-\not p_{2}+m_{Q}\right) \gamma_{\alpha}\left(\not p_{1}+\not k+m_{Q}\right) \gamma_{\beta} u_{Q}\left(p_{1}\right) \\
& \bar{u}_{q}\left(p_{3}\right) \gamma^{\beta}\left(\not p_{3}+\not k+m_{q}\right) \gamma^{\alpha} v_{q}\left(p_{4}\right) \\
& \frac{1}{k^{2}\left(p_{1}+p_{2}+k-q\right)^{2}\left[\left(q-p_{2}\right)^{2}-m_{Q}^{2}\right]\left[\left(p_{1}+k\right)^{2}-m_{Q}^{2}\right]\left[\left(p_{3}+k\right)^{2}-m_{q}^{2}\right]}, \quad(A 2), \\
M_{C} & =\int \frac{d^{4} k}{(2 \pi)^{4}} \varepsilon^{* \mu}(\gamma) \bar{v}_{Q}\left(p_{2}\right)\left(-i g_{s} T^{a} \gamma_{\alpha}\right) \frac{i}{p_{1}-\not q+\not k-m_{Q}}\left(-i e Q_{Q} \gamma_{\mu}\right) \\
& \frac{i}{p_{1}+\not k-m_{Q}}\left(-i g_{s} T^{b} \gamma_{\beta}\right) u_{Q}\left(p_{1}\right) \\
& \overline{u_{q}}\left(p_{3}\right)\left(-i g_{s} T^{b} \gamma^{\beta}\right) \frac{i}{p_{3}+\not k-m_{q}}\left(-i g_{s} T^{a} \gamma^{\alpha}\right) v_{q}\left(p_{4}\right) \\
& \frac{-i}{\left(p_{1}+p_{2}+k-q\right)^{2}} \frac{-i}{k^{2}} \\
& =e Q_{Q} g_{s}^{4} T^{a} T^{b} \otimes T^{b} T^{a} \int \frac{d^{4} k}{(2 \pi)^{4}} \overline{v_{Q}}\left(p_{2}\right) \gamma_{\alpha}\left(p_{1}-\not q+\not k+m_{Q}\right) \gamma_{\mu}\left(p_{1}+\not k+m_{Q}\right) \gamma_{\beta} u_{Q}\left(p_{1}\right) \\
& \overline{u_{q}}\left(p_{3}\right) \gamma^{\beta}\left(\not p_{3}+\not k+m_{q}\right) \gamma^{\alpha} v_{q}\left(p_{4}\right) \varepsilon^{* \mu}(\gamma) \\
& \frac{1}{k^{2}\left(p_{1}+p_{2}+k-q\right)^{2}\left[\left(p_{1}-q+k\right)^{2}-m_{Q}^{2}\right]\left[\left(p_{1}+k\right)^{2}-m_{Q}^{2}\right]\left[\left(p_{3}+k\right)^{2}-m_{q}^{2}\right]}, \quad(A 3) .
\end{aligned}
$$

The expressions for the process $J / \psi \rightarrow \rho^{0} \gamma$ are

$$
\begin{aligned}
& M_{A}=\frac{i \pi^{2} e Q_{Q} g_{s}^{4} T^{a} T^{b} \otimes T^{b} T^{a}}{\left(\left(\frac{1}{2} p_{V}-q\right)^{2}-m_{Q}^{2}\right)(2 \pi)^{4}} \varepsilon^{* \mu}(\gamma) \\
& {\left[D_{1}^{\rho \nu}\left(x, m_{q}\right)\left(\frac{1}{2} p_{V}-q\right)^{\sigma} \overline{O_{1 \mu \sigma \rho \nu}^{4}}+D_{1}^{\rho \nu}\left(x, m_{q}\right) m_{Q} \overline{O_{2 \mu \rho \nu}^{4}}+D_{2}^{\nu}\left(x, m_{q}\right)\left(\frac{1}{2} p_{V}-q\right)^{\sigma} m_{Q} \overline{O_{3 \mu \nu \sigma}^{4}}\right.} \\
& \left.+D_{2}^{\nu}\left(x, m_{q}\right) m_{Q}^{2} \overline{O_{4 \mu \nu}^{4}}\right]
\end{aligned}
$$

where

$$
\begin{aligned}
& \overline{O_{\mu \sigma \rho \nu}^{1}}=2 g_{\nu \sigma} \bar{v}_{Q} \gamma_{\mu} u_{Q} \bar{u}_{q} \gamma_{\rho} v_{q}+2 g_{\sigma \mu} \bar{v}_{Q} \gamma_{\nu} u_{Q} \overline{u_{q}} \gamma_{\rho} v_{q}-2 g_{\mu \nu} \bar{v}_{Q} \gamma_{\sigma} u_{Q} \bar{u}_{q} \gamma_{\rho} v_{q} \\
& +2 g_{\rho \nu} \bar{v}_{Q} \gamma_{\mu} u_{Q} \bar{u}_{q} \gamma_{\sigma} v_{q}+2 g_{\rho \nu} g_{\sigma \mu} \bar{v}_{Q} \gamma_{\beta} u_{Q} \bar{u}_{q} \gamma^{\beta} v_{q}-2 g_{\rho \nu} \bar{v}_{Q} \gamma_{\sigma} u_{Q} \bar{u}_{q} \gamma_{\mu} v_{q} \\
& \overline{O_{\mu \rho \nu}^{2}}=2 i \bar{v}_{Q} \sigma_{\nu \mu} u_{Q} \bar{u}_{q} \gamma_{r h o} v_{q}+2 i g_{\rho \nu} \bar{v}_{Q} \sigma_{\beta \mu} u_{Q} \bar{u}_{q} \gamma^{\beta} v_{q} \\
& \overline{O_{\mu \nu \sigma}^{3}}=2 i \bar{v}_{Q} \sigma_{\sigma \mu} u_{Q} \bar{u}_{q} \gamma_{\nu} v_{q} \\
& \overline{O_{\mu \nu}^{4}}=2 \bar{v}_{Q} \gamma_{\mu} u_{Q} \bar{u}_{q} \gamma_{\nu} v_{q} . \\
M_{B}= & \frac{i \pi^{2} e Q_{Q} g_{s}^{4} T^{a} T^{b} \otimes T^{b} T^{a}}{\left(\left(\frac{1}{2} p_{V}-q\right)^{2}-m_{Q}^{2}\right)(2 \pi)^{4}} \varepsilon^{* \mu}(\gamma)
\end{aligned}
$$




$$
\begin{aligned}
& {\left[-D_{1}^{\rho \nu}\left(x, m_{q}\right)\left(\frac{1}{2} p_{V}-q\right)^{\sigma} \overline{O_{\mu \sigma \rho \nu}^{1}}+D_{1}^{\rho \nu}\left(x, m_{q}\right) m_{Q} \overline{O_{\mu \rho \nu}^{2}}+D_{2}^{\nu}\left(x, m_{q}\right)\left(\frac{1}{2} p_{V}-q\right)^{\sigma} m_{Q} \overline{O_{\mu \nu \sigma}^{3}}\right.} \\
& \left.-D_{2}^{\nu}\left(x, m_{q}\right) m_{Q}^{2} \overline{O_{\mu \nu}^{4}}\right] .
\end{aligned}
$$

The corresponding hadronic matrix elements are

$$
\begin{aligned}
& \left\langle V^{\prime}\left|\bar{q} \gamma_{\alpha} q \sum_{i}\left(C_{i}\left(x, m_{q}\right) \overline{O_{i}}\right) \bar{Q} \gamma_{\beta} Q\right| V\right\rangle \\
& =i f_{V^{\prime}} \varepsilon_{V^{\prime} \alpha} m_{V^{\prime}} \int_{0}^{1} d x \phi_{V^{\prime}}(\mu, x) \sum_{i}\left(C_{i}\left(x, m_{q}\right) \overline{O_{i}}\right) i f_{V} \varepsilon_{V} M_{V} \\
& \left\langle V^{\prime}\left|\bar{q} \gamma_{\alpha} q \sum_{i}\left(C_{i}\left(x, m_{q}\right) \overline{O_{i}}\right) \bar{Q} \sigma_{\beta \rho} Q\right| V\right\rangle \\
& =i f_{V^{\prime}} \varepsilon_{V^{\prime} \alpha} m_{V^{\prime}} \int_{0}^{1} d x \phi_{V^{\prime}}(\mu, x) \sum_{i}\left(C_{i}\left(x, m_{q}\right) \overline{O_{i}}\right) i f_{V} i\left(\varepsilon_{V \beta} p_{V} \rho-p_{V \beta} \varepsilon_{V} \rho\right) .
\end{aligned}
$$

Concretely the hadronic matrix elements of $M_{A}$ and $M_{B}$ are

$$
\begin{aligned}
& \left\langle V^{\prime} \gamma\left|M_{A}\right| V\right\rangle \\
& =i \pi^{2} e Q_{Q} g_{s}^{4} T^{a} T^{b} \otimes T^{b} T^{a} \frac{1}{(2 \pi)^{4}} \frac{1}{\left(\frac{1}{2} p_{V}-q\right)^{2}-m_{Q}^{2}} \frac{1}{N_{c}^{2}} \varepsilon^{* \mu}(\gamma) \int_{0}^{1} d x \phi_{P}(\mu, x) \\
& \left\{i f _ { V ^ { \prime } } m _ { V ^ { \prime } } i f _ { V } M _ { V } \left[2 g_{\nu \sigma} \varepsilon_{V \mu} \varepsilon_{V^{\prime} \rho}+2 g_{\sigma \mu} \varepsilon_{V^{\prime}} \varepsilon_{V^{\prime} \rho}-2 g_{\mu \nu} \varepsilon_{V} \varepsilon_{V^{\prime} \rho}+2 g_{\rho \nu} \varepsilon_{V} \varepsilon_{V^{\prime} \sigma}\right.\right. \\
& \left.+2 g_{\rho \nu} g_{\sigma \mu} \varepsilon_{V} \varepsilon_{V^{\prime}}^{\beta}-2 g_{\rho \nu} \varepsilon_{V} \varepsilon_{V^{\prime} \mu}\right] \sum_{q=u, d, s}\left(D_{1}^{\rho \nu}\left(x, m_{q}\right)\right)\left(\frac{1}{2} p_{V}-q\right)^{\sigma} \\
& +i f_{V^{\prime}} m_{V^{\prime}} i f_{V} i\left[2 i\left(\varepsilon_{V \nu} p_{V \mu}-p_{V \nu} \varepsilon_{V \mu}\right) \varepsilon_{V^{\prime} \rho}+2 i g_{\rho \nu}\left(\varepsilon_{V} \beta p_{V \mu}-p_{V} \varepsilon_{V \mu}\right) \varepsilon_{V^{\prime}}^{\beta}\right] \sum_{q=u, d, s}\left(D_{1}^{\rho \nu}\left(x, m_{q}\right)\right) m_{Q} \\
& +i f_{V^{\prime}} m_{V^{\prime}} i f_{V} i\left[2 i\left(\varepsilon_{V \sigma} p_{V \mu}-p_{V \sigma} \varepsilon_{V}\right) \varepsilon_{V^{\prime}{ }^{\prime}}\right] \sum_{q=u, d, s}\left(D_{2}^{\nu}\left(x, m_{q}\right)\right)\left(\frac{1}{2} p_{V}-q\right)^{\sigma} m_{Q} \\
& \left.+i f_{V^{\prime}} m_{V^{\prime}} i f_{V} M_{V}\left[2 \varepsilon_{V \mu} \varepsilon_{V^{\prime} \nu_{\nu}}\right] \sum_{q=u, d, s}\left(D_{2}^{\nu}\left(x, m_{q}\right)\right) m_{Q}^{2},\right\}
\end{aligned}
$$

and

$$
\begin{aligned}
& \left\langle V^{\prime} \gamma\left|M_{B}\right| V\right\rangle \\
& =i \pi^{2} e Q_{Q} g_{s}^{4} T^{a} T^{b} \otimes T^{b} T^{a} \frac{1}{(2 \pi)^{4}} \frac{1}{\left(\frac{1}{2} p_{V}-q\right)^{2}-m_{Q}^{2}} \frac{1}{N_{c}^{2}} \varepsilon^{* \mu}(\gamma) \int_{0}^{1} d x \phi_{P}(\mu, x) \\
& \left\{-i f_{V^{\prime}} m_{V^{\prime}} i f_{V} M_{V}\left[2 g_{\nu \sigma} \varepsilon_{V \mu^{\mu}} \varepsilon_{V^{\prime} \rho}+2 g_{\sigma \mu} \varepsilon_{V \nu^{\nu}} \varepsilon_{V^{\prime} \rho}-2 g_{\mu \nu} \varepsilon_{V \sigma^{\sigma}} \varepsilon_{V^{\prime} \rho}+2 g_{\rho \nu} \varepsilon_{V \mu^{\mu}} \varepsilon_{V^{\prime} \sigma}\right.\right. \\
& \left.+2 g_{\rho \nu} g_{\sigma \mu} \varepsilon_{V} \varepsilon_{V^{\prime}}^{\beta}-2 g_{\rho \nu} \varepsilon_{V} \varepsilon_{V^{\prime} \mu}\right] \sum_{q=u, d, s}\left(D_{1}^{\rho \nu}\left(x, m_{q}\right)\right)\left(\frac{1}{2} p_{V}-q\right)^{\sigma} \\
& +i f_{V^{\prime}} m_{V^{\prime}} i f_{V} i\left[2 i\left(\varepsilon_{V \nu} p_{V \mu}-p_{V^{\nu}} \varepsilon_{V} \mu\right) \varepsilon_{V^{\prime} \rho}+2 i g_{\rho \nu}\left(\varepsilon_{V} \beta p_{V}^{\mu}-p_{V} \varepsilon_{V} \mu\right) \varepsilon_{V^{\prime}}^{\beta}\right] \sum_{q=u, d, s}\left(D_{1}^{\rho \nu}\left(x, m_{q}\right)\right) m_{Q} \\
& +i f_{V^{\prime}} m_{V^{\prime}} i f_{V} i\left[2 i\left(\varepsilon_{V} p_{V \mu}-p_{V} \varepsilon_{V} \mu\right) \varepsilon_{V^{\prime} \nu}\right] \sum_{q=u, d, s}\left(D_{2}^{\nu}\left(x, m_{q}\right)\right)\left(\frac{1}{2} p_{V}-q\right)^{\sigma} m_{Q}
\end{aligned}
$$




$$
\left.-i f_{V^{\prime}} m_{V^{\prime}} i f_{V} M_{V}\left[2 \varepsilon_{V} \varepsilon_{V^{\prime}{ }_{\nu}}\right] \sum_{q=u, d, s}\left(D_{2}^{\nu}\left(x, m_{q}\right)\right) m_{Q}^{2} \cdot\right\}
$$

\section{Appendix B}

The two four-point loop functions in our text are:

$$
\begin{aligned}
& D_{1}^{\rho \nu}\left(x, m_{q}\right)=\frac{1}{i \pi^{2}} \int d^{4} k \frac{\left(p_{1}+k\right)^{\rho}\left(p_{2}+k\right)^{\nu}}{k^{2}\left[\left(p_{1}+k\right)^{2}-m_{Q}^{2}\right]\left(k+p_{2}\right)^{2}\left[\left(k+p_{3}\right)^{2}-m_{q}^{2}\right]} \\
& D_{2}^{\nu}\left(x, m_{q}\right)=\frac{1}{i \pi^{2}} \int d^{4} k \frac{\left(p_{3}+k\right)^{\nu}}{k^{2}\left[\left(p_{1}+k\right)^{2}-m_{Q}^{2}\right]\left(k+p_{2}\right)^{2}\left[\left(k+p_{3}\right)^{2}-m_{q}^{2}\right]}
\end{aligned}
$$

with

$$
p_{1}=\frac{1}{2} p_{V}, p_{2}=p_{V}-q, p_{3}=p_{P} x .
$$

And the three five-point loop functions are:

$$
\begin{aligned}
& E_{1}^{\nu \rho \sigma}\left(x, m_{q}\right)=\frac{1}{i \pi^{2}} \int d^{4} k \frac{k^{\nu} k^{\rho} p_{3}^{\sigma}+k^{\nu} p_{1}^{\rho}\left(k+p_{3}\right)^{\sigma}+p_{4}^{\nu}\left(k+p_{1}\right)^{\rho}\left(k+p_{3}\right)^{\sigma}}{k^{2}\left[\left(k+p_{3}\right)^{2}-m_{q}^{2}\right]\left(k+p_{2}\right)^{2}\left[\left(k+p_{4}\right)^{2}-m_{Q}^{2}\right]\left[\left(k+p_{1}\right)^{2}-m_{Q}^{2}\right]} \\
& E_{2}^{\nu \sigma}\left(x, m_{q}\right)=\frac{1}{i \pi^{2}} \int d^{4} k \frac{\left(k+p_{4}\right)^{\nu}\left(k+p_{3}\right)^{\sigma}}{k^{2}\left[\left(k+p_{3}\right)^{2}-m_{q}^{2}\right]\left(k+p_{2}\right)^{2}\left[\left(k+p_{4}\right)^{2}-m_{Q}^{2}\right]\left[\left(k+p_{1}\right)^{2}-m_{Q}^{2}\right]} \\
& E_{3}^{\rho \sigma}\left(x, m_{q}\right)=\frac{1}{i \pi^{2}} \int d^{4} k \frac{\left(k+p_{1}\right)^{\rho}\left(k+p_{3}\right)^{\sigma}}{k^{2}\left[\left(k+p_{3}\right)^{2}-m_{q}^{2}\right]\left(k+p_{2}\right)^{2}\left[\left(k+p_{4}\right)^{2}-m_{Q}^{2}\right]\left[\left(k+p_{1}\right)^{2}-m_{Q}^{2}\right]}
\end{aligned}
$$

with

$$
p_{1}=\frac{1}{2} p_{V}, p_{2}=p_{V}-q, p_{3}=p_{P} x, p_{4}=\frac{1}{2} p_{V}-q,
$$

which can be decomposed into somes of four-point loop functions according to [4].

$$
\begin{aligned}
& E_{1}^{\nu \rho \sigma}\left(x, m_{q}\right)=\left(\sum_{i=0}^{4} \frac{\operatorname{det}\left(Y_{i}\right)}{\operatorname{det}(Y)} D^{(f i n) \nu \rho}(i)+\sum_{i, j=1}^{4}(-1)^{i+j} \frac{\operatorname{det}\left(\hat{Z}_{i j}^{(4)}\right)}{\operatorname{det}(Y)} 2 p_{j \alpha} \mathcal{D}^{\alpha \nu \rho}(i)\right) p_{3}^{\sigma} \\
& +\left(\sum_{i=0}^{4} \frac{\operatorname{det}\left(Y_{i}\right)}{\operatorname{det}(Y)} D^{(f i n) \nu \sigma}(i)+\sum_{i, j=1}^{4}(-1)^{i+j} \frac{\operatorname{det}\left(\hat{Z}_{i j}^{(4)}\right)}{\operatorname{det}(Y)} 2 p_{j \alpha} \mathcal{D}^{\alpha \nu \sigma}(i)\right) p_{1}^{\rho} \\
& +\left(\sum_{i=0}^{4} \frac{\operatorname{det}\left(Y_{i}\right)}{\operatorname{det}(Y)} D^{(f i n) \nu}(i)+\sum_{i, j=1}^{4}(-1)^{i+j} \frac{\operatorname{det}\left(\hat{Z}_{i j}^{(4)}\right)}{\operatorname{det}(Y)} 2 p_{j \alpha} \mathcal{D}^{\alpha \nu}(i)\right) p_{1}^{\rho} p_{3}^{\sigma} \\
& +\left(\sum_{i=0}^{4} \frac{\operatorname{det}\left(Y_{i}\right)}{\operatorname{det}(Y)} D^{(f i n) \rho \sigma}(i)+\sum_{i, j=1}^{4}(-1)^{i+j} \frac{\operatorname{det}\left(\hat{Z}_{i j}^{(4)}\right)}{\operatorname{det}(Y)} 2 p_{j \alpha} \mathcal{D}^{\alpha \rho \sigma}(i)\right) p_{4}^{\nu} \\
& +\left(\sum_{i=0}^{4} \frac{\operatorname{det}\left(Y_{i}\right)}{\operatorname{det}(Y)} D^{(f i n) \rho}(i)+\sum_{i, j=1}^{4}(-1)^{i+j} \frac{\operatorname{det}\left(\hat{Z}_{i j}^{(4)}\right)}{\operatorname{det}(Y)} 2 p_{j \alpha} \mathcal{D}^{\alpha \rho}(i)\right) p_{4}^{\nu} p_{3}^{\sigma} \\
& +\left(\sum_{i=0}^{4} \frac{\operatorname{det}\left(Y_{i}\right)}{\operatorname{det}(Y)} D^{(f i n) \sigma}(i)+\sum_{i, j=1}^{4}(-1)^{i+j} \frac{\operatorname{det}\left(\hat{Z}_{i j}^{(4)}\right)}{\operatorname{det}(Y)} 2 p_{j \alpha} \mathcal{D}^{\alpha \sigma}(i)\right) p_{4}^{\nu} p_{1}^{\rho}
\end{aligned}
$$




$$
\begin{aligned}
& +\left(\sum_{i=0}^{4} \frac{\operatorname{det}\left(Y_{i}\right)}{\operatorname{det}(Y)} D_{0}^{(f i n)}(i)+\sum_{i, j=1}^{4}(-1)^{i+j} \frac{\operatorname{det}\left(\hat{Z}_{i j}^{(4)}\right)}{\operatorname{det}(Y)} 2 p_{j \alpha} \mathcal{D}^{\alpha}(i)\right) p_{4}^{\nu} p_{1}^{\rho} p_{3}^{\sigma}, \\
& E_{2}^{\nu \sigma}\left(x, m_{q}\right)=\left(\sum_{i=0}^{4} \frac{\operatorname{det}\left(Y_{i}\right)}{\operatorname{det}(Y)} D^{(f i n) \nu \sigma}(i)+\sum_{i, j=1}^{4}(-1)^{i+j} \frac{\operatorname{det}\left(\hat{Z}_{i j}^{(4)}\right)}{\operatorname{det}(Y)} 2 p_{j \alpha} \mathcal{D}^{\alpha \nu \sigma}(i)\right) \\
& +\left(\sum_{i=0}^{4} \frac{\operatorname{det}\left(Y_{i}\right)}{\operatorname{det}(Y)} D^{(f i n) \nu}(i)+\sum_{i, j=1}^{4}(-1)^{i+j} \frac{\operatorname{det}\left(\hat{Z}_{i j}^{(4)}\right)}{\operatorname{det}(Y)} 2 p_{j \alpha} \mathcal{D}^{\alpha \nu}(i)\right) p_{3}^{\sigma} \\
& +\left(\sum_{i=0}^{4} \frac{\operatorname{det}\left(Y_{i}\right)}{\operatorname{det}(Y)} D^{(f i n) \sigma}(i)+\sum_{i, j=1}^{4}(-1)^{i+j} \frac{\operatorname{det}\left(\hat{Z}_{i j}^{(4)}\right)}{\operatorname{det}(Y)} 2 p_{j \alpha} \mathcal{D}^{\alpha \sigma}(i)\right) p_{4}^{\nu} \\
& +\left(\sum_{i=0}^{4} \frac{\operatorname{det}\left(Y_{i}\right)}{\operatorname{det}(Y)} D_{0}^{(f i n)}(i)+\sum_{i, j=1}^{4}(-1)^{i+j} \frac{\operatorname{det}\left(\hat{Z}_{i j}^{(4)}\right)}{\operatorname{det}(Y)} 2 p_{j \alpha} \mathcal{D}^{\alpha}(i)\right) p_{4}^{\nu} p_{3}^{\sigma}, \\
& E_{3}^{\rho \sigma}\left(x, m_{q}\right)=\left(\sum_{i=0}^{4} \frac{\operatorname{det}\left(Y_{i}\right)}{\operatorname{det}(Y)} D^{(f i n) \rho \sigma}(i)+\sum_{i, j=1}^{4}(-1)^{i+j} \frac{\operatorname{det}\left(\hat{Z}_{i j}^{(4)}\right)}{\operatorname{det}(Y)} 2 p_{j \alpha} \mathcal{D}^{\alpha \rho \sigma}(i)\right) \\
& +\left(\sum_{i=0}^{4} \frac{\operatorname{det}\left(Y_{i}\right)}{\operatorname{det}(Y)} D^{(f i n) \rho}(i)+\sum_{i, j=1}^{4}(-1)^{i+j} \frac{\operatorname{det}\left(\hat{Z}_{i j}^{(4)}\right)}{\operatorname{det}(Y)} 2 p_{j \alpha} \mathcal{D}^{\alpha \rho}(i)\right) p_{3}^{\sigma} \\
& +\left(\sum_{i=0}^{4} \frac{\operatorname{det}\left(Y_{i}\right)}{\operatorname{det}(Y)} D^{(f i n) \sigma}(i)+\sum_{i, j=1}^{4}(-1)^{i+j} \frac{\operatorname{det}\left(\hat{Z}_{i j}^{(4)}\right)}{\operatorname{det}(Y)} 2 p_{j \alpha} \mathcal{D}^{\alpha \sigma}(i)\right) p_{1}^{\rho} \\
& +\left(\sum_{i=0}^{4} \frac{\operatorname{det}\left(Y_{i}\right)}{\operatorname{det}(Y)} D_{0}^{(f i n)}(i)+\sum_{i, j=1}^{4}(-1)^{i+j} \frac{\operatorname{det}\left(\hat{Z}_{i j}^{(4)}\right)}{\operatorname{det}(Y)} 2 p_{j \alpha} \mathcal{D}^{\alpha}(i)\right) p_{1}^{\rho} p_{3}^{\sigma}, \\
& \left.+Y^{(f i n}\right)
\end{aligned}
$$

with :

$$
\begin{aligned}
& (Y)_{i j}=m_{i}^{2}+m_{j}^{2}-\left(p_{i}^{\prime}-p_{j}^{\prime}\right)^{2}, \quad i, j=0, \ldots, 4 \\
& \left(\hat{Z}^{(4)}\right)_{k l}=2 p_{k}^{\prime} p_{l}^{\prime}, \quad k, l=1, \ldots, 4 \\
& m_{0}=0, m_{1}=m_{q}, m_{2}=0, m_{3}=m_{Q}, m_{4}=m_{Q} \\
& p_{0}^{\prime}=0, p_{1}^{\prime}=p_{3}, p_{2}^{\prime}=p_{4}, p_{3}^{\prime}=p_{2}, p_{4}^{\prime}=p_{1} .
\end{aligned}
$$

And $D^{(f i n) \nu \rho}(i)$ 's denote the ultraviolet-finite four-point functions that are obtained by removing the $i$ th propagator in the five-point functions. Let us take $D^{(f i n) \nu \rho}(1)$ as an example, and it is:

$$
D^{(f i n) \nu \rho}(1)=\frac{1}{i \pi^{2}} \int d^{4} k \frac{k^{\nu} k^{\rho}}{k^{2}\left(k+p_{2}\right)^{2}\left[\left(k+p_{4}\right)^{2}-m_{Q}^{2}\right]\left[\left(k+p_{1}\right)^{2}-m_{Q}^{2}\right]}
$$

where only four factors exist at the denominator.

$Y_{i}$ is obtained from the 5-dimensional Cayley matrix $Y$ by replacing all entries in the $i$ th column with 1 and the 3 -dimensional matrices $\hat{Z}_{i j}^{(4)}$ result from the 4 -dimensional Gram matrix $\hat{Z}^{(4)}$ by discarding the $i$ th row and $j$ th column. 


\section{References}

[1] S. Okubo, Phys.Lett. 5 (1963) 163; G. Zweig, CERN Report No. 8182/TH 401; G. Zweig, CERN Rep.8419/TH-412 (1964); J. Iizuka, Prog.Theor.Phys. Suppl. 37-8 (1966) 21.

[2] F. Close, G. Farrar and Z. Li, Phys.Rev. D55 (1997) 5749; F. Close and Q. Zhao, Phys.Lett. B586 (2004) 332.

[3] J.G. Körner, J.H. Kühn, M. Krammer and H. Schneider, Nucl.Phys. B229 (1983) 115-141.

[4] A. Denner, S. Dittmaier, Nucl.Phys. B658 (2003) 175-202; A. Denner, Nucl.Phys. B519 (1998) 39-84.

[5] G.'t Hooft and M. Veltman, Nucl. Phys. B153 (1979) 365-401; A. Denner, U. Nierste and R. Scharf, Nucl. Phys. B367 (1991) 637-656.

[6] Y.D. Yang, hep-ph/0404018.

[7] C.S. Huang, Commun. Theor. Phys. 3 (1984) 203.

[8] S. Eidelman, et al., Particle Data Group, Phys.Lett. B592 (2004) 1.

[9] T. Hahn, hep-ph/9905354.

[10] M. Krammer, Phys.Lett. B74 (1978) 381; B. Guberina and J.H. Kühn, Nuovo Cim.Lett. 32 (1981) 295; J.H.Kühn, Proc.Workshop on Doris experiments, DESY,10-11 February 1981; J.H. Kühn, J. Kaplan and E.G.O. Safiani, Nucl.Phys. B157 (1979) 125.

[11] V.L. Chernyak, A.R. Zhitnitsky, Nucl.Phys. B201 (1996) 2182-2193.

[12] N.H. Fuchs, M.D. Scadron, Phys.Rev. D20 (1979) 2421.

[13] M. Beneke, G. Buchalla, M. Neubert and C.T. Sachrajda, Nucl.Phys. B591 (2000) 313-418.

[14] V.A. Novikov, M.A. Shifman, A.I. Vainstein and V.I. Zakharov, Nucl.Phys. B165 (1980) 55; T. Feldman and P. Kroll, Phys.Scripta, T99 (2002),13.

[15] J.P. Ma, Phys.Rev. D65 (2002) 097506.

[16] N. Brambilla et al., hep-ph/0412158, ${ }^{1}$

[17] S. Chung, Phys.Rev. D48 (1993) 1225.

[18] A. Deandrea, G. Nardulli and A.D. Polosa, hep-ph/0211431; A. Deandrea, G. Nardulli and A.D. Polosa, Phys.Rev. D68 (2003) 034002, hep-ph/0302273.

[19] P. Ball, V.M. Braun, Phys.Rev. D54 (1996) 2182.

[20] H. Cheng, K. Yang, Phys.Rev. D63 (2001) 074011.

[21] J.P. Ma, Nucl.Phys. B605 (2001) 625-643.

\footnotetext{
${ }^{1}$ We would like to thank Dr. Brambilla, who reminds us to read this report which will appear in the yellow book of CERN.
} 
[22] D. Ebert, R.N. Faustov and V.O. Galkin, hep-ph/0308150 S. Fleming and A.K. Leibovich, Phys.Rev. D67 (2003) 074035, hep-ph/0212094; A. Ali and A. Parkhomenko, Eur Phys.J. C30 (2003) 183-195, hep-ph/0304278 H. Dong, X. Guo, X. Li, R. Zhang, Phys.Rev. D57 (1998) 6807-6813, hep-ph/9805277.

[23] B.I. Ermolaev, M. Greco and S.I. Troyan, hep-ph/0106317; C.-H. Chang, X. Wu, Eur.Phys.J. C38 (2004) 267-276, hep-ph/0309121; H. Fritzsch and Y. Zhou, Phys.Rev. D68 (2003) 034015, hep-ph/0301038. 
Figure 1: Radiative decays of a ${ }^{3} S_{1}(Q \bar{Q})$ bound-state into ${ }^{1} S_{0}(q \bar{q})$ states in the lowest order.

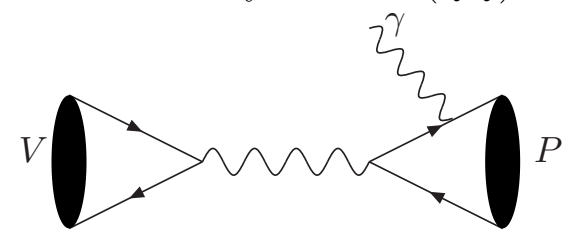

(a)

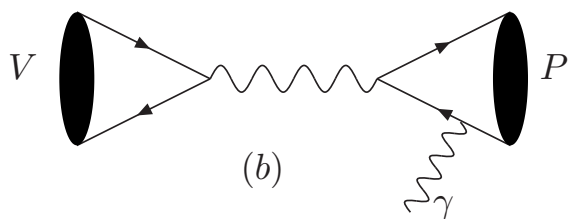

(b)

Figure 2: Radiative decays of a ${ }^{3} S_{1}(Q \bar{Q})$ bound-state into ${ }^{1} S_{0}(q \bar{q})$ states in the next order.

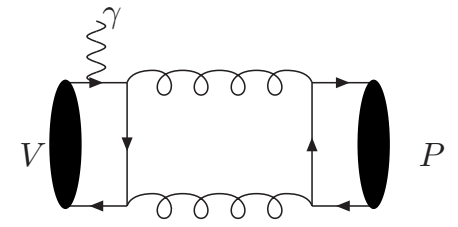

(a)

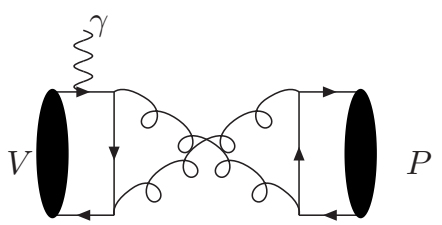

(d)

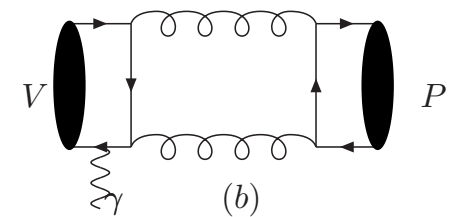

(b)

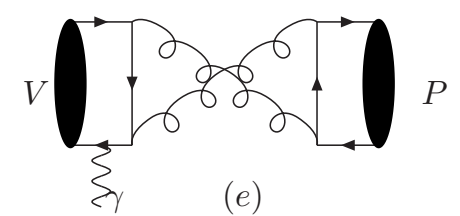

(e)

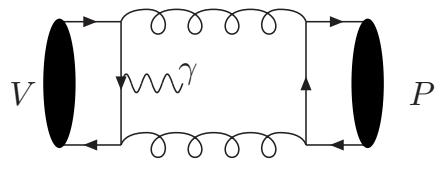

(c)

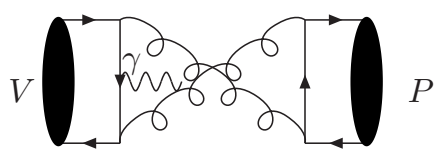

(f) 